\title{
$\Rightarrow$ ASTHMA AND ALLERGY
}

\section{A fibrinogen root to airway inflammation}

The molecular mechanisms that underlie allergic inflammatory diseases remain unclear and this is an area of active debate. Reporting in Science, Millien et al. now suggest that hyperactivation of an antifungal pathway involving fibrinogen cleavage and Toll-like receptor 4 (TLR4) signalling contributes to allergic airway inflammation.

Wild-type mice that were intranasally challenged daily for 2 weeks with a fungal proteinase derived from Aspergillus oryzae (PAO) developed canonical features of asthma, including airway hyperresponsiveness, eosinophilia, enhanced mucin $5 \mathrm{AC}$ (Muc5ac) expression, airway goblet cell hyperplasia and production of

\section{exogenous and endogenous proteinases can cleave fibrinogen to generate TLR4 ligands that prime innate immune cells to respond to IL-13}

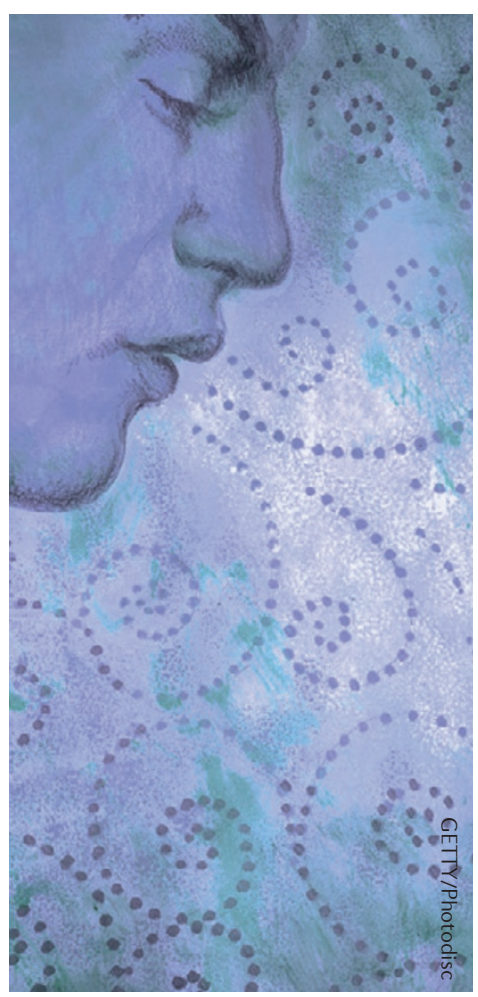

the $\mathrm{T}$ helper $2\left(\mathrm{~T}_{\mathrm{H}} 2\right)$ cell-associated cytokines interleukin-4 (IL-4), IL-5 and IL-13.

By contrast, Tlr4 $4^{-/-}$mice challenged with PAO showed reduced or attenuated disease symptoms, but equivalent levels of IL-4 production compared with wild-type mice. Similarly, the induction of allergic lung inflammation, but not IL-4 production, in response to allergen challenge with proteinase-free ovalbumin or with the conidia of Aspergillus niger was TLR4 dependent. These data indicate that TLR4 is required for the development of allergic airway inflammation, irrespective of allergen proteinase content, but not for IL-4 production in the lungs.

Alveolar macrophages from PAO-treated mice had increased TLR4-dependent expression of several genes that are associated with antifungal immunity. In addition, $\mathrm{PAO}$-activated bone marrow-derived macrophages (BMDMs) from wildtype mice, but not from $\mathrm{Tlr} 4^{-/-}$mice, controlled fungal growth (known as fungistasis) when the conidia of A. niger were added to the cell cultures. Of note, this fungistatic activity only occurred in the presence of fetal bovine serum. These data suggest that PAO functions through both a serum factor and TLR4 to induce macrophage antifungal immunity.

Further investigations showed that stimulation of BMDMs with the endogenous proteinase thrombin, which converts the serum factor fibrinogen to fibrin, resulted in similar fungistatic activity to that observed after PAO stimulation. Furthermore, fibrinogen cleavage products (FCPs) - generated by incubation of fibrinogen with PAO or thrombin - induced fungistasis when added to BMDM cultures without fetal bovine serum. Moreover, the proteinase inhibitor hirudin reduced the fungistatic activity of PAOstimulated BMDMs in the presence of fetal bovine serum. Interestingly, FCPs also induced TLR4-dependent MUC5AC and IL-13 receptor- $\alpha 1$ (IL-13Ra1) expression, as well as fungistatic activity, by ex vivo airway epithelial cells. These data suggest that exogenous and endogenous proteinases can cleave fibrinogen to generate TLR4 ligands that prime epithelial cells to respond to IL-13.

But does this pathway have a role in allergic airway disease? Mice challenged with high-dose FCPs showed modest eosinophil recruitment and Muc5ac expression in the lungs but not airway hyperresponsiveness or IL-4 production. Furthermore, the inclusion of hirudin during allergen challenge greatly reduced PAOinduced airway hyperresponsiveness, eosinophilia, Muc5ac expression and IL-13 production. Thus, both FCP-TLR4 signalling and cytokine signalling from $\mathrm{T}_{\mathrm{H}} 2$ cells are required for the full induction of allergic airway disease.

Taken together, these data suggest that overwhelming exposure to endogenous or exogenous proteinases may lead to hyperactivation of an antifungal pathway and may drive allergic airway inflammation through both fibrinogen and TLR4dependent and TLR4-independent pathways.

Olive Leavy

ORIGINAL RESEARCH PAPER Millien, V. O. et al Cleavage of fibrinogen by proteinases elicits allergic responses through Toll-like receptor 4. Science 341, 792-796 (2013) 